Article

\title{
Turbulence Models for Single Phase Flow Simulation of Cyclonic Flotation Columns
}

\author{
Shiqi Meng ${ }^{1}$, Xiaoheng Li ${ }^{1}$, Xiaokang Yan ${ }^{1} * \mathbb{E}$, Lijun Wang ${ }^{2}$, Haijun Zhang ${ }^{3}$ and Yijun Cao ${ }^{3, *}$ \\ 1 School of Chemical Engineering and Technology, China University of Mining and Technology, \\ Xuzhou 221116, China \\ 2 School of Power Engineering, China University of Mining and Technology, Xuzhou 221116, China \\ 3 Chinese National Engineering Research Center of Coal Preparation and Purification, Xuzhou 221116, China \\ * Correspondence: xk-yan@cumt.edu.cn (X.Y.); yijuncao@126.com (Y.C.)
}

Received: 10 June 2019; Accepted: 25 July 2019; Published: 30 July 2019

\begin{abstract}
Cyclonic fields are important for cyclonic static microbubble flotation columns (FCSMCs), one of the most important developments in column flotation technology, particularly for separation of fine particles, where the internal flow field has enormous influence on flotation performance. PIV (particle image velocimetry) and CFD (computational fluid dynamics) are the most effective methods to study flow fields. However, data is insufficient for FCSMC flow fields and similar cyclonic equipment, with turbulence model simulations producing different views to measured data. This paper employs an endoscope and PIV to measure axial and cross sections for single-phase swirling flow fields in FCSMCs. We then compare various turbulence model simulations (Reynolds stress model (RSM), standard $k-\varepsilon$, realizable $k-\varepsilon$, and RNG (renormalization group) $k-\varepsilon$ ) to the measured data. The RSM (Reynolds stress model) predicts cyclonic flow field best in flotation columns with $16.22 \%$ average relative velocity deviation. Although the realizable $k-\varepsilon$ model has less than $30 \%$ relative deviation in radial and tangential directions, axial deviations reach $78.11 \%$. Standard $k-\varepsilon$ and RNG $k-\varepsilon$ models exhibited approximately $40 \%$ and $30 \%$ radial and tangential deviation, respectively, and cannot be used even for trend predictions for axial velocity. $k-\varepsilon$ models are based on isotropic assumptions with semi-empirical formulas summarized from experiments, whereas RSM fundamentally considers laminar flow and Reynolds stress, and hence is more suitable for anisotropic performance. This study will contribute to flotation column and other cyclonic flow field equipment research.
\end{abstract}

Keywords: CFD; cyclonic flow field; flotation column; endoscopic laser PIV; turbulence models

\section{Introduction}

Cyclonic structures are employed in cyclonic static microbubble flotation columns (FCSMCs), which have sprung up since it was patented in 1999 [1] and been successfully applied to refining stages, to facilitate centrifugal separation [2], bubble dispersion [3], and the reduction of sorting granularity [4]. Figure 1 shows a typical FCSMC structure, incorporating an inverted cone in its middle part. Air is introduced and crushed into microbubbles by bubble generators in the pipe flow. The mixture of slurry and bubbles enters the flotation column from two symmetrical inlets on the swirl inverted cone, forming a swirl flow field. Hydrophobic mineral particles attach to the bubbles and are carried up to be concentrated. Under the centrifugal force in the swirling flow field, the high-density and coarse particles move outward and are discharged from the tail port. The remaining minerals enter the mid port and are separated again in the following pipe flow unit. This complex process involve lift, drag, and other interaction since gas and particles move in liquid. Under liquid forces and interface forces, collisions, adhesions, and detachments between particles and bubbles happen, as shown in Figure 2. Generally, this process happened in a turbulence flow environment [5] and are affected by the turbulent 
flow field. Therefore, the turbulence in cyclonic flow fields is an important research topic for this type of flotation column and other similar equipment.

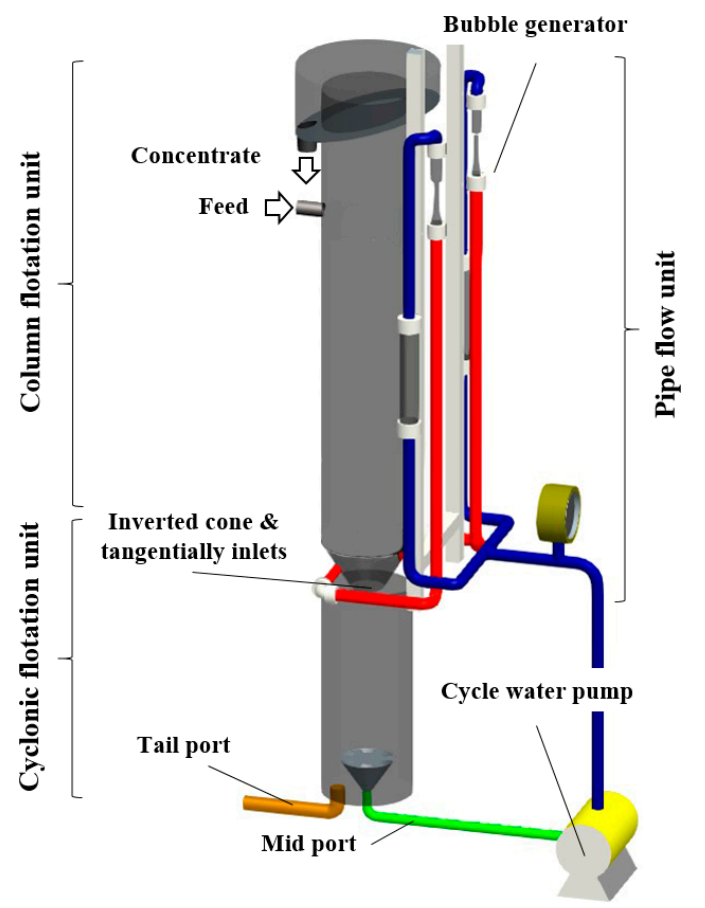

Figure 1. Typical cyclonic static microbubble flotation column structure.

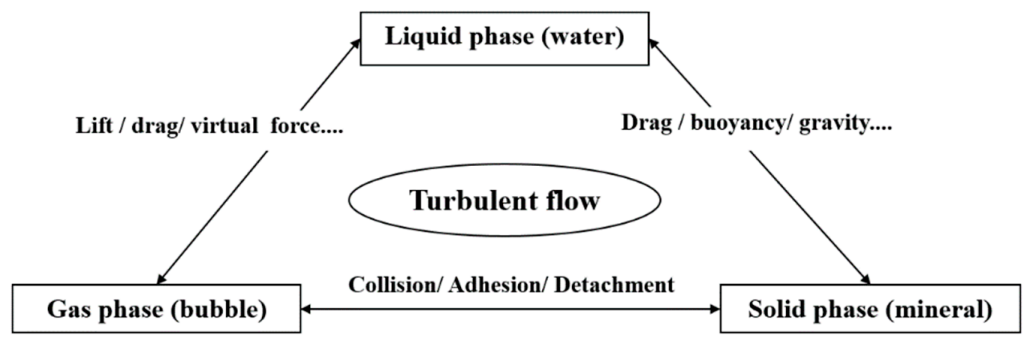

Figure 2. Forces and interactions inside an operating flotation column.

Fine mineral particles and water are thoroughly mixed in a stirred tank to form a homogeneous slurry, and then introduced into the flotation column. Flotation feed particles are generally $<0.074 \mathrm{~mm}$ in diameter, and flow well in the slurry. This work will provide an important reference for the study of multiphase flow in a flotation column and will have a positive impact on the industrial application of flotation columns.

Flow fields have been studied for many years with continuous developments in experimental fluid dynamics (EFD) and computational fluid dynamics (CFD). Particle image velocimetry (PIV) is an advanced EFD-based velocity measurement method developed in the late 1970s. PIV can record velocity distribution information for a large number of spatial points in the same transient state, providing flow field structure and characteristics [6].

Although PIV has been widely used for cyclonic flow field measurement, data remains inadequate to fully reflect detailed flow features. For example, Marins et al. [7] used PIV to measure axial and tangential velocity of a hydrocyclone without an air core to obtain the radial velocity. Cui et al. [8] used PIV to measure axial and radial velocity in the axial section of a hydrocyclone with an air core, but cross-section measurement was not possible due to overflow. Tangential velocity is particularly difficult to measure for flotation columns since the column top is open and the swirling flow interferes with the light path. Yan et al. [9] and Wang et al. [10] used PIV to measure single-phase cyclonic flow 
fields for flotation columns, but only obtained axial velocity by measuring one quasi axial section of the flotation column under a fixed volume flow rate.

Computational fluid dynamics provides effective methods to numerically study flow fields and has been successfully used to calculate various cyclonic equipment parameters. However, numerical simulations tend to simplify boundary conditions and material properties, and discretization differences can lead to different results. Therefore, numerical simulation requires experimental data to verify its reliability. Reasonable numerical simulation has a guiding effect on experimental and theoretical analysis, which can make up for the lack of experimental work. The turbulence model selection has significant influence on CFD simulation accuracy for single-phase cyclonic flow. Due to the large resources occupied by DNS (direct numerical simulation) and LES (large eddy simulation), the Reynolds stress model (RSM) and $k-\varepsilon$ turbulence models are more commonly used for column simulation. Khan et al. [11] proposed a three-dimensional (3D) model of a bubble column simulated using $k-\varepsilon$ and RSM models. They performed LDA (laser doppler anemometry measurements) in the column to verify the simulation, and confirmed that $k-\varepsilon$ and RSM models could effectively predict bubble column internal flow fields. However, several issues remain for application of these turbulence models to cyclonic flow simulations. Wang et al. [10] compared single-phase PIV experiment results with CFD predictions, and concluded that the RSM model was more suitable for single-phase cyclonic field simulation in a FCSMC. Zhang et al. [12] and Yan et al. [13] used the standard $k-\varepsilon$ turbulence model to simulate single-phase internal flow fields within flotation columns and analyze their benefits. Swain et al. [14] used RSM and standard $k-\varepsilon$ models to simulate gas-liquid two-phase flow in a hydrocyclone, and concluded that both models could predict the hydrocyclone flow pattern. Azadi et al. [15] used RNG $k-\varepsilon$ and Reynolds stress models to calculate cyclonic flow fields, and showed that RSM predictions produced smaller deviations but required higher computational power than the RNG $k-\varepsilon$ model.

Two main problems have prevented flow field application to cyclonic flotation columns: Measured data is not available for the tangential section and turbulent numerical models remain uncertain.

Therefore, the current study first improved the traditional PIV experimental method for flotation columns, and then developed an experimental platform for measuring the swirling field axial and cross sections inside a flotation column, providing detailed flow field information including axial, radial and tangential velocity. We subsequently performed 3D and unsteady single-phase simulations for cyclonic flotation columns, and investigated different turbulence model suitability to accurately model single-phase flow fields inside the flotation column. This study provides a new reference for numerical studies and PIV tests on flotation columns and similar equipment flow fields.

\section{PIV Experiment}

\subsection{Experiment Apparatus}

We constructed a cyclonic flotation column as sown in Figure 1 for PIV tests, with height $=$ $1400 \mathrm{~mm}$ and inner diameter $=190 \mathrm{~mm}$. The flotation column was constructed from glass to ensure transparency. The experimental medium was deionized water at room temperature. A water-filled cube box surrounded the column flotation unit to eliminate cylindrical surface refraction. Water from the pump was divided into two symmetrical lines. After passing through a bubble generator with the air inlet closed, water tangentially flowed into the inverted cone, forming a cyclonic field. Since the single-phase experiment did not include particles, we neglected feeding and discharging effects.

\subsection{Experiment Method}

We measured flotation column axial and cross sections to obtain axial, radial, and tangential velocity. The flotation column position was fixed during the experiment, and measurements were performed by adjusting the relative positions of the laser light sheet and CCD camera. The scanning surface formed by the laser light sheet should be kept perpendicular to the imaging direction of the 
CCD camera. The tracer particles used for the PIV test were hollow glass spheres having a diameter of $10 \mu \mathrm{m}$, and its density was $1.05 \mathrm{~g} / \mathrm{cm}^{3}$, which shows good water following performance. The software used for the PIV test was Dynamic Studio, which is a mature image system software platform for many fluid mechanics experiments, such as PIV, LIF, combustion, and atomization analysis. In the PIV test, Dynamic Studio processed image information into vectors, velocity, and other hydrodynamic parameters of the flow field.

Figure 3 shows how the laser light sheet, CCD camera, and test sections were placed to measure the flotation column axial section. Several bubbles clustered near the column axis, which would interfere with the laser light sheet, reduce the flow field and luminance at the axis and in the other half of the flotation column, impacting measurement accuracy. This approach is similar to that employed by Wang et al. [10]. Therefore, following some initial experiments, we adopted the quasi-axial section, deviating $10 \mathrm{~mm}$ from the central axial section as the measurement plane to ensure more accurate experimental data, as shown in the dash-solid section in Figure 3 (260-340 mm above the inverted cone). The left side was illuminated by laser with sufficient intensity. Four circulating flow types were selected for experiments, considering industrial applications. Table 1 shows optimal experimental parameters derived from several trial experiments and adjustments, and we averaged 200 photograph pairs. Experimental data at $300 \mathrm{~mm}$ height was extracted for comparison with CFD simulations.

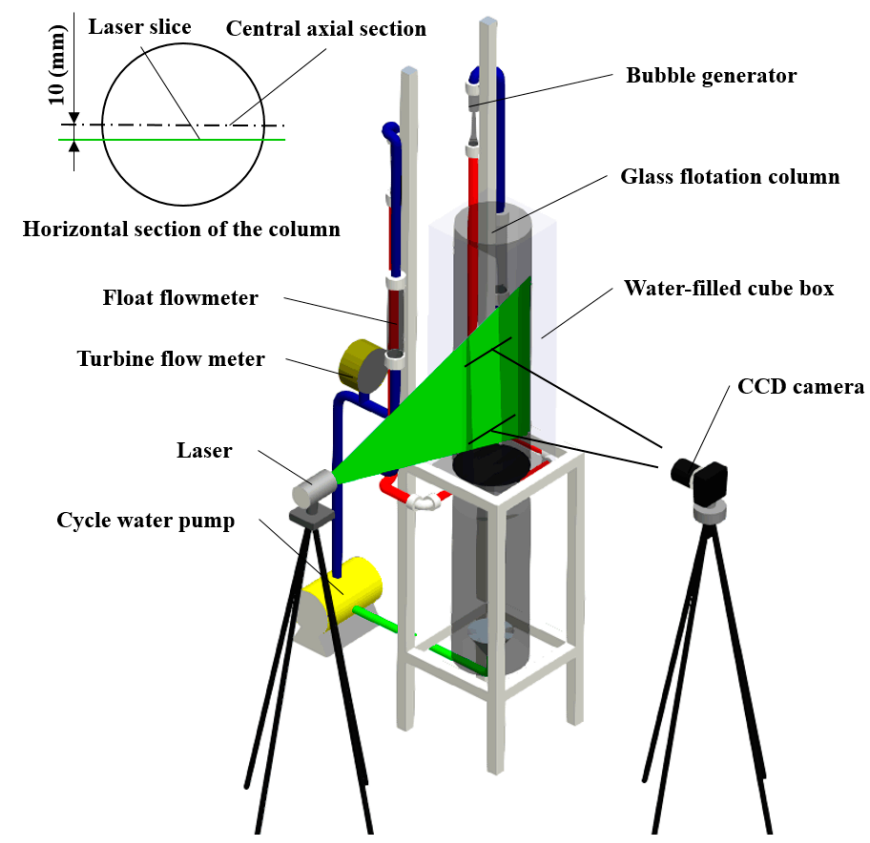

Figure 3. Particle image velocimetry (PIV) measurement of a quasi-axial section.

Table 1. PIV experimental parameters for cyclonic static microbubble flotation column vertical sections.

\begin{tabular}{ccccc}
\hline $\begin{array}{c}\text { Measuring } \\
\text { Surface }\end{array}$ & $\begin{array}{c}\text { Recycling Flow } \\
\left(\mathbf{m}^{3} / \mathbf{h}\right)\end{array}$ & $\begin{array}{c}\text { Measurement } \\
\text { Parameters }\end{array}$ & Laser Interval $(\boldsymbol{\mu s})$ & $\begin{array}{c}\text { Trigger } \\
\text { Frequency }\end{array}$ \\
\hline Vertical section, & 0.25 & Axial velocity, & 1200 & \\
$\begin{array}{c}\text { 260-340 mm above } \\
\text { the cyclonic }\end{array}$ & 0.50 & radial velocity & 600 & $9.0 \mathrm{~Hz}$ \\
flotation unit & 1.00 & 300 & \\
\hline
\end{tabular}

To obtain tangential velocity, we installed the CCD camera above the column, providing downward looking images to measure the horizontal section. However, the water swirls when the flotation column is in operation, and the free surface between air and water inside the column flotation zone, forms an inverted cone. Laser-illuminated tracer particles are refracted when passing through this free surface, causing deviations in the CCD images. To solve this problem, we employed an endoscope for 
the PIV test, allowing the lens to be inserted into the water to test the flow field, and hence eliminating most optical interference. Velarde [16] and Helmi [17] used an endoscopic laser PIV technique for dense gas-solid fluidized beds in 2016 and 2018, respectively, which inspired the current proposed PIV measurement system for cyclonic flow fields in the flotation column.

Figure 4 shows how an endoscope was installed onto the CCD camera and extended below the free surface. The measured horizontal section was $300 \mathrm{~mm}$ above the inverted cone. Table 2 shows optimal experimental parameters derived from many preliminary experiments, with 200 photograph pairs captured for averaging. Similar to flotation column axial section measurements discussed above, we selected data along a straight line in the radial direction, and the same four circulating flows were employed, as shown in Table 2.

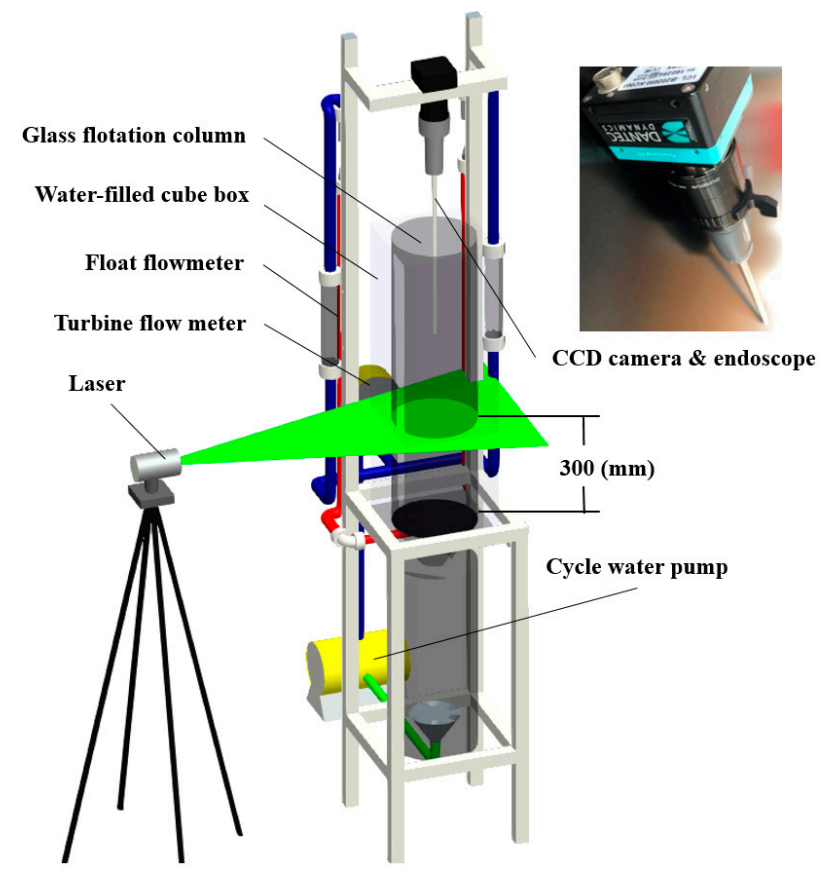

Figure 4. Horizontal section PIV measurement.

Table 2. Cyclonic static microbubble flotation column (FCSMC) horizontal section PIV measurement parameters.

\begin{tabular}{ccccc}
\hline Measuring Surface & $\begin{array}{c}\text { Recycling Flow } \\
\left(\mathbf{m}^{3} / \mathbf{h}\right)\end{array}$ & $\begin{array}{c}\text { Measurement } \\
\text { Parameter }\end{array}$ & $\begin{array}{c}\text { Laser } \\
\text { Interval }(\boldsymbol{\mu s})\end{array}$ & $\begin{array}{c}\text { Trigger } \\
\text { Frequency }\end{array}$ \\
\hline Column cross-section, & 0.25 & & 1000 & \\
300 mm above the & 0.50 & Tangential velocity & 600 & $9.0 \mathrm{~Hz}$ \\
column flotation unit & 1.00 & & 400 & \\
\hline
\end{tabular}

\section{Numerical Simulation}

\subsection{Turbulence Models}

Turbulence models greatly impact results from single-phase numerical simulations (Table 3). Turbulence model expression establishment and application differ, producing inconsistent predictions for many flow field types. We compared the RSM, standard $k-\varepsilon$ model, realizable $k-\varepsilon$ model, and RNG $k-\varepsilon$ models. As one of the most widely used turbulence models, the standard $k-\varepsilon$ model is based on transport equations for turbulent kinetic energy, k, and its dissipation rate, $\varepsilon$ [18], which are defined as

$$
\frac{\partial(\rho k)}{\partial t}+\frac{\partial\left(\rho k u_{i}\right)}{\partial x_{i}}=\frac{\partial}{\partial x_{j}}\left[\left(\mu+\frac{\mu_{t}}{\sigma_{k}}\right) \frac{\partial k}{\partial x_{j}}\right]+G_{k}+G_{b}-\rho \varepsilon-Y_{M}+S_{k},
$$




$$
\frac{\partial(\rho \varepsilon)}{\partial t}+\frac{\partial\left(\rho \varepsilon u_{i}\right)}{\partial x_{i}}=\frac{\partial}{\partial x_{j}}\left[\left(\mu+\frac{\mu_{t}}{\sigma_{\varepsilon}}\right) \frac{\partial \varepsilon}{\partial x_{j}}\right]+\frac{C_{1 \varepsilon} \varepsilon}{k}\left(G_{k}+C_{3 \varepsilon} G_{b}\right)-C_{2 \varepsilon} \rho \frac{\varepsilon^{2}}{k}+S_{\varepsilon} .
$$

Table 3. Symbol and interpretation.

\begin{tabular}{cccc}
\hline Symbol & Interpretation & Symbol & Interpretation \\
\hline$k$ & Turbulence kinetic energy & $\varphi$ & Press strain \\
$\varepsilon$ & Turbulence dissipation rate & $D$ & Turbulence diffusion \\
$\rho$ & Density & $p$ & Pressure \\
$u$ & Velocity & $\mu$ & Viscosity \\
$C$ & Constant & $t$ & Time \\
$P$ & Turbulence production term & $\delta$ & Kronecker delta function \\
$i, 1$ & $x$ component & $j, 2$ & $y$ component \\
$k, 3$ & $z$ component (in the RSM) & $S_{k}, S_{\varepsilon}$ & User-defined source terms. \\
$G_{k}$ & The generation of turbulence kinetic energy due to the mean velocity gradients, calculated as \\
$G_{b}$ & described in Modeling Turbulent Production in the $k$ - $\varepsilon$ Models. \\
$Y_{M}$ & The generation of turbulence kinetic energy due to buoyancy, calculated as described in Effects \\
$\sigma_{k}, \sigma_{\varepsilon}$ & of Buoyancy on Turbulence in the $k-\varepsilon$ Models. & The contribution of the fluctuating dilatation in compressible turbulence to the overall dissipation \\
\hline
\end{tabular}

The RNG $k-\varepsilon$ model was proposed by Yakhot and Orzga [19] in 1986. It is similar in form to the standard $k-\varepsilon$ model, but includes some refinements. The RNG model has an additional term in its equation that improves the accuracy for rapidly strained flows. In addition, the effect of swirl on turbulence is included in the model, enhancing accuracy for swirling flows. What is more, it provides an analytical formula for turbulent Prandtl numbers, while the standard $k-\varepsilon$ model uses user-specified, constant values. Therefore, these features make the RNG $k-\varepsilon$ model more accurate and reliable for a wider class of flows than the standard $k-\varepsilon$ model. The equations are given as:

$$
\begin{gathered}
\frac{\partial(\rho k)}{\partial t}+\frac{\partial\left(\rho k u_{i}\right)}{\partial x_{i}}=\frac{\partial}{\partial x_{j}}\left[a_{k} \mu_{e f f} \frac{\partial k}{\partial x_{j}}\right]+G_{k}+G_{b}-\rho \varepsilon-Y_{M}+S_{k \prime} \\
\frac{\partial(\rho \varepsilon)}{\partial t}+\frac{\partial\left(\rho \varepsilon u_{i}\right)}{\partial x_{i}}=\frac{\partial}{\partial x_{j}}\left(a_{\varepsilon} \mu_{e f f} \frac{\partial \varepsilon}{\partial x_{j}}\right)+\frac{C_{1 \varepsilon} \varepsilon}{k}\left(G_{k}+C_{3 \varepsilon} G_{b}\right)-C_{2 \varepsilon} \rho \frac{\varepsilon^{2}}{k}-R_{\varepsilon}+S_{\varepsilon} .
\end{gathered}
$$

The realizable $k-\varepsilon$ model was proposed by Moin et al. [20] who believe that the standard $k-\varepsilon$ model may cause stress errors when the time-averaged strain rate is strong. The model [21] implements an alternative formulation for turbulent viscosity [22] based on a modified transport equation for dissipation derived from an exact equation for mean-square vorticity fluctuation transportation:

$$
\begin{gathered}
\frac{\partial(\rho k)}{\partial t}+\frac{\partial\left(\rho k u_{j}\right)}{\partial x_{j}}=\frac{\partial}{\partial x_{j}}\left[\left(\mu+\frac{\mu_{t}}{\sigma_{k}}\right) \frac{\partial k}{\partial x_{j}}\right]+G_{k}+G_{b}-\rho \varepsilon-Y_{M}+S_{k}, \\
\frac{\partial(\rho \varepsilon)}{\partial t}+\frac{\partial\left(\rho \varepsilon u_{j}\right)}{\partial x_{j}}=\frac{\partial}{\partial x_{j}}\left[\left(\mu+\frac{\mu_{t}}{\sigma_{\varepsilon}}\right) \frac{\partial \varepsilon}{\partial x_{j}}\right]+\rho C_{1} E \varepsilon-\rho C_{2} \frac{\varepsilon^{2}}{k+\sqrt{v \varepsilon}}+C_{1 \varepsilon} \frac{\varepsilon}{k} C_{3 \varepsilon} G_{b}+S_{\varepsilon} .
\end{gathered}
$$

RSM closes the Reynolds-averaged Naiver-Stokes equations by abandoning the isotropic eddy-viscosity hypothesis and solving transport equations for Reynolds stresses, together with an equation for the dissipation rate [23-25]. The control equations are as follows:

Continuity equations:

$$
\frac{\partial \rho}{\partial t}+\frac{\partial}{\partial}\left(\rho u_{i}\right)=0
$$


Momentum equation:

$$
\frac{\partial u_{i}}{\partial t}+\frac{\partial}{\partial x_{j}}\left(u_{i} u_{j}\right)=-\frac{1}{\rho} \frac{\partial p}{\partial x_{i}}+\frac{1}{\rho} \frac{\partial}{\partial x_{j}}\left[\mu\left(\frac{\partial u_{i}}{\partial x_{j}}+\frac{\partial u_{j}}{\partial x_{i}}\right)\right]+\frac{\partial}{\partial x_{j}}\left(-\overline{u_{i}^{\prime} u_{j}^{\prime}}\right)
$$

where

$$
u_{i}=\overline{u_{i}}+u_{i}^{\prime} .
$$

Turbulence production term $P_{i j}$ is defined as

$$
P_{i j}=\rho\left(\overline{u_{i}^{\prime} u_{k}^{\prime}} \frac{\partial u_{j}}{\partial x_{k}}+\overline{u_{j} u_{k}^{\prime}} \frac{\partial u_{j}}{\partial x_{k}}\right)
$$

Turbulence dissipation rate $\varepsilon_{i j}$ is given as

$$
\varepsilon_{i j}=2 \mu \overline{\frac{\partial u_{i}^{\prime}}{\partial x_{k}} \frac{\partial u_{j}^{\prime}}{\partial x_{k}}}
$$

Press strain $\varphi_{i j}$ is defined as

$$
\varphi_{i j}=\overline{p\left(\frac{\partial u_{i}^{\prime}}{\partial x_{j}}+\frac{\partial u_{j}^{\prime}}{\partial x_{i}}\right)}
$$

Turbulence diffusion $D_{T, i j}$ is given as

$$
D_{T, i j}=-\frac{\partial}{\partial x_{k}}\left(\rho \overline{u_{i}^{\prime} u_{j}^{\prime} u_{k}^{\prime}}+\overline{p u_{i}^{\prime}} \delta_{j k}-\mu \frac{\partial}{\partial x_{k}} \overline{u_{i}^{\prime} u_{j}^{\prime}}\right)
$$

\subsection{Geometry and Mesh}

The 3D FCSMC model was established for the experimental device, as shown in Figure 5, and geometric model processing was consistent with Wang et al. [10] and Yan et al. [13]. A structured mesh was constructed aside from the inverted cone, which was unstructured mesh (see Figure 5), and grid independence was verified. Figure 6 shows axial velocity along the horizontal direction at $300 \mathrm{~mm}$ column height for different mesh schemes. We selected a model incorporating 379,658 grids.

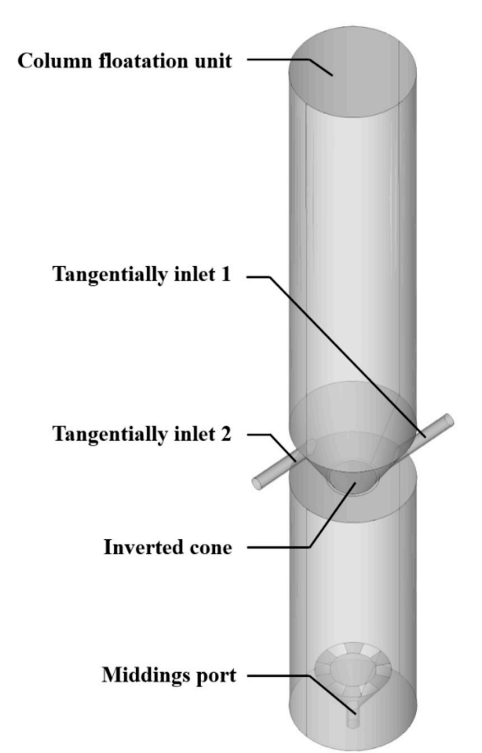

(a)

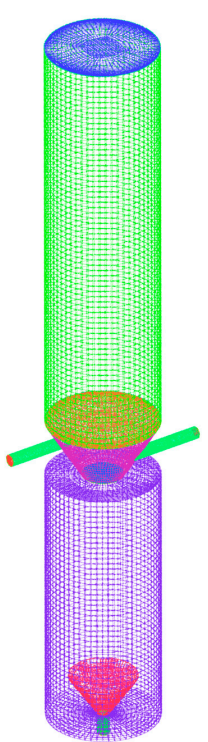

(b)

Figure 5. Flotation column 3D model (a) and mesh (b). 


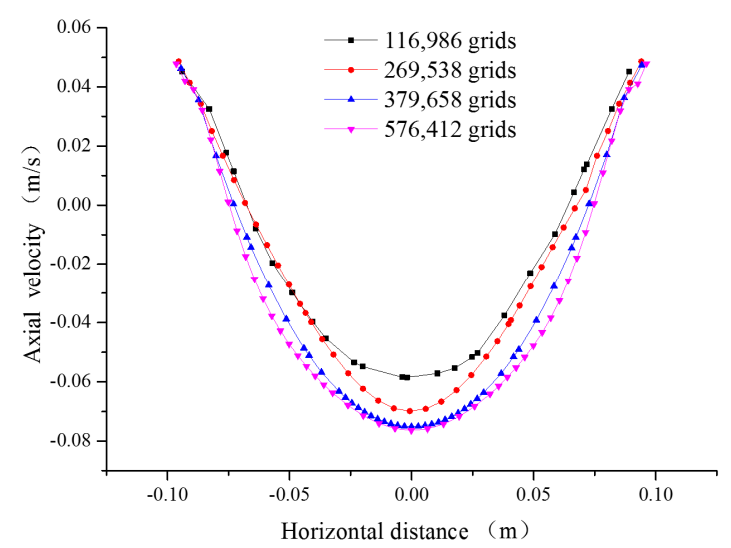

Figure 6. Grid independence: The curve of axial velocity along the horizontal direction on a section $300 \mathrm{~mm}$ above the inverted cone at $1.50 \mathrm{~m}^{3} / \mathrm{h}$ flux.

\subsection{Boundary Conditions and Solution}

In order to match the numerical simulation with the PIV experiment, we set the boundary conditions according to the experiment. Two tangential inlets (Figure 5, Inlet 1 and 2) in the cyclonic unit are defined as velocity inlets, with fluid speeds of $0.11,0.22,0.44$, and $0.66 \mathrm{~m} / \mathrm{s}$ for $0.25,0.50,1.00$, and $1.50 \mathrm{~m}^{3} / \mathrm{h}$ flow rates, respectively. Since the fluid was single phase, we assumed that all feed was discharged from the middling port, with the amount of feed equal to the amount of discharge. Therefore, the middling port outlet was defined as a negative velocity inlet with $0.22,-0.44,-0.88$, and $-1.32 \mathrm{~m} / \mathrm{s}$, respectively. The acceleration of gravity is $9.81 \mathrm{~m} / \mathrm{s}$, which is vertically downward along the axis. Table 4 shows all boundary conditions and calculation values.

Table 4. Circulation flow boundary conditions.

\begin{tabular}{cccccc}
\hline \multirow{2}{*}{ Boundary } & \multirow{2}{*}{ Type } & \multicolumn{4}{c}{ Circulating Volume Rate $\left(\mathbf{m}^{\mathbf{3}} / \mathbf{h}\right)$} \\
\cline { 3 - 6 } & & $\mathbf{0 . 2 5}$ & $\mathbf{0 . 5 0}$ & $\mathbf{1 . 0 0}$ & $\mathbf{1 . 5 0}$ \\
\hline Inlet 1 & Inlet velocity $(\mathrm{m} / \mathrm{s})$ & 0.11 & 0.22 & 0.44 & 0.66 \\
Inlet 2 & Inlet velocity $(\mathrm{m} / \mathrm{s})$ & 0.11 & 0.22 & 0.44 & 0.66 \\
Concentrate outlet & Symmetry & - & - & - & - \\
Middling port outlet & Inlet velocity $(\mathrm{m} / \mathrm{s})$ & -0.22 & -0.44 & -0.88 & -1.32 \\
\hline
\end{tabular}

Commercial CFD code ANSYS Fluent was employed to carry out the simulation. We used the SIMPLE algorithm for pressure-velocity coupling, the least squares cell based on gradient, the PRESTO! scheme for pressure discretization, and the second order upwind scheme for momentum discretization, turbulent kinetic energy, turbulent dissipation rate, and Reynolds stresses. The calculation used an unsteady solver, hence once the parameters and residuals of the monitored point reached statistical stability, the results of the previous $20 \mathrm{~s}$ duration were averaged and used for subsequent analysis.

\subsection{CFD and PIV Comparison}

\subsubsection{Velocity Vectors}

Figure 7a shows the vertical PIV experimental vectors located at the plane $260-340 \mathrm{~mm}$ above the inverted cone and parallel to the $10 \mathrm{~mm}$ axial section. Simulation and PIV results were compared at $1.00 \mathrm{~m}^{3} / \mathrm{s}$ to verify prediction accuracy. PIV showed gradually increasing water vector magnitude as distance from the column wall increased, followed by a sharp reduction, with water flowing approximately horizontally toward the axis. Figure $7 \mathrm{~b}$ shows that RSM model vectors were consistent with the PIV results, whereas other models (Figure 7c-e) produced divergent directions close to the axis, being diagonally downwards.

Figure 8 compares PIV (Figure $8 \mathrm{a}$ ) and model (Figure $8 \mathbf{b}-\mathrm{e}$ ) results for the horizontal plane $300 \mathrm{~mm}$ above the inverted cone. All the models are consistent with PIV in that the water rotates counterclockwise around the center. 


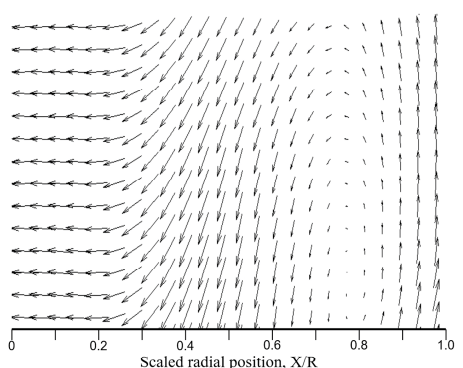

(a) PIV measured water flow vectors

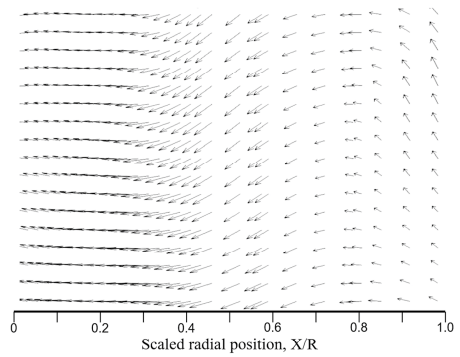

(b) RSM model flow vectors

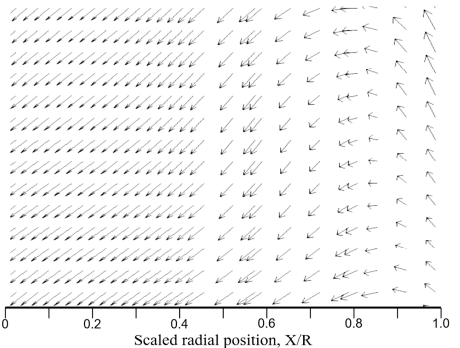

(c) Standard $k-\varepsilon$ model flow vectors
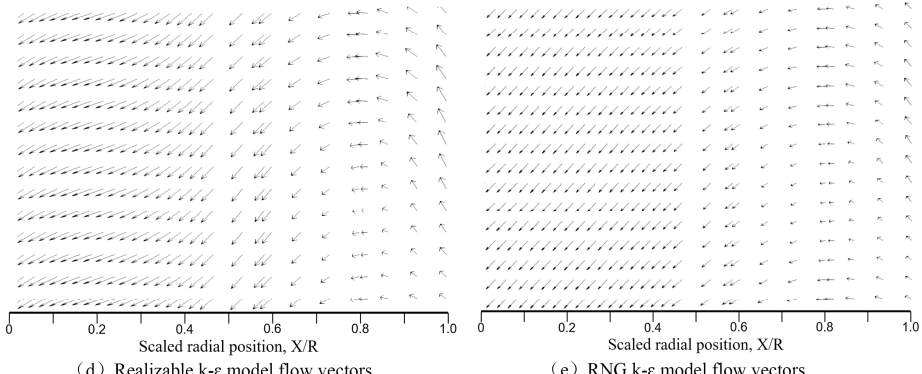

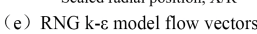

(d) Realizable $k-\varepsilon$ model flow vector

Figure 7. Experimental and simulation water flow vectors in quasi-axis sections. (a) PIV measured water flow vectors; (b) RSM model flow vectors; (c) Standard $k-\varepsilon$ model flow vectors; (d) Realizable $k-\varepsilon$ model flow vectors; (e) RNG $k-\varepsilon$ model flow vectors.

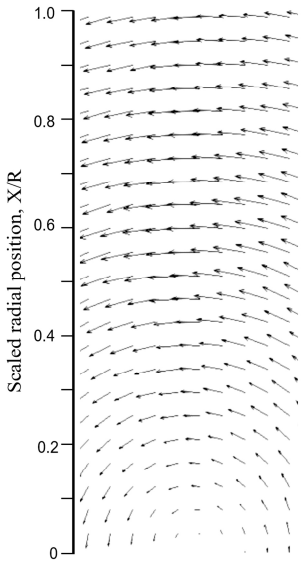

(a) PIV measured water flow vectors

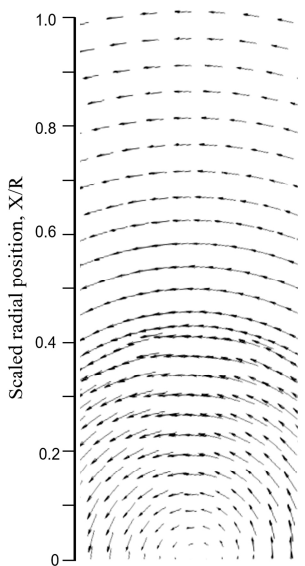

(b) RSM model flow vectors

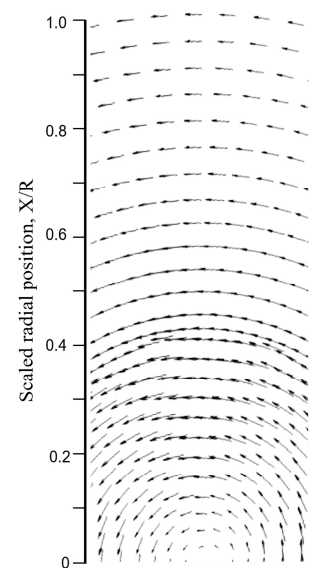

(c) Standard k- $\varepsilon$ model flow vectors

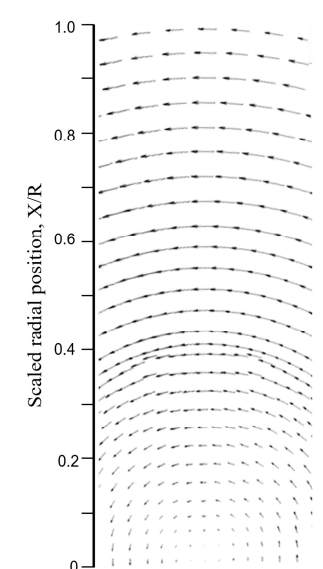

(d) Realizable k- $\varepsilon$ model flow vectors

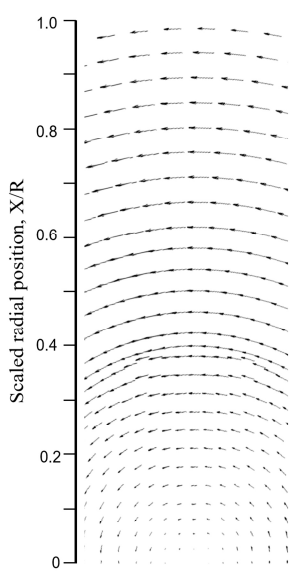

(e) RNG k- $\varepsilon$ model flow vectors

Figure 8. Experiment model water flow vectors in horizontal sections. (a) PIV measured water flow vectors; (b) RSM model flow vectors; (c) Standard $k-\varepsilon$ model flow vectors; (d) Realizable $k-\varepsilon$ model flow vectors; (e) RNG $k-\varepsilon$ model flow vectors. 


\subsubsection{Quantitative Analysis}

Figures 9 and 10 compare the simulated and measured velocity profiles along the horizontal direction for a cyclic flux of $0.25,0.50,1.00$, and $1.50 \mathrm{~m}^{3} / \mathrm{h}$, respectively, at $z=300 \mathrm{~mm}, 10 \mathrm{~mm}$ off-axis. As for Figure 11, the velocity distribution of the simulation and measurement in the horizontal direction from the axis to the wall at $z=300 \mathrm{~mm}$ is compared. The RSM and realizable $k-\varepsilon$ turbulent model axial velocity distribution trends exhibit the in " $\sqrt{ }$ " shape, consistent with PIV results. However, the standard $k-\varepsilon$ and RNG $k-\varepsilon$ model trends diverge widely.

Figures 10 and 11 show radial and tangential velocity results, respectively. Distribution trends along the horizontal direction are consistent with PIV results for all turbulence models.

Thus, RSM and realizable $k-\varepsilon$ models exhibit good prediction performance for velocity distributions in all directions, whereas the standard $k-\varepsilon$ model and the RNG $k-\varepsilon$ model can only predict velocity distribution trends in radial and tangential directions.

Tables 5-7 summarize absolute deviations between simulation and PIV results (excluding data within 3 $\mathrm{mm}$ of the zero velocity point) for axial, radial, and tangential velocities, respectively. Since the standard $k-\varepsilon$ model and the RNG $k$ - $\varepsilon$ model cannot predict velocity distribution trends in the axial direction, only radial and tangential errors were analyzed. The RSM model achieved the smallest error for all directions.

Table 5. Simulation model axial velocity absolute deviation from PIV measurement.

\begin{tabular}{ccccc}
\hline \multirow{2}{*}{ Flow Rate $\left(\mathbf{m}^{\mathbf{3}} \mathbf{h}\right)$} & \multicolumn{4}{c}{ Turbulence Model } \\
\cline { 2 - 5 } & RSM (\%) & Standard $k-\varepsilon \mathbf{\%})$ & Realizable $\boldsymbol{k - \varepsilon} \mathbf{( \% )}$ & RNG $\boldsymbol{k - \varepsilon} \mathbf{~ ( \% )}$ \\
\hline 0.25 & 19.89 & 87.12 & 106.15 & 102.86 \\
0.50 & 18.76 & 71.87 & 68.42 & 80.11 \\
1.00 & 14.39 & 90.42 & 67.22 & 86.85 \\
1.50 & 16.39 & 83.58 & 70.65 & 88.26 \\
Mean absolute deviation & 17.36 & 83.25 & 78.11 & 89.52 \\
\hline
\end{tabular}
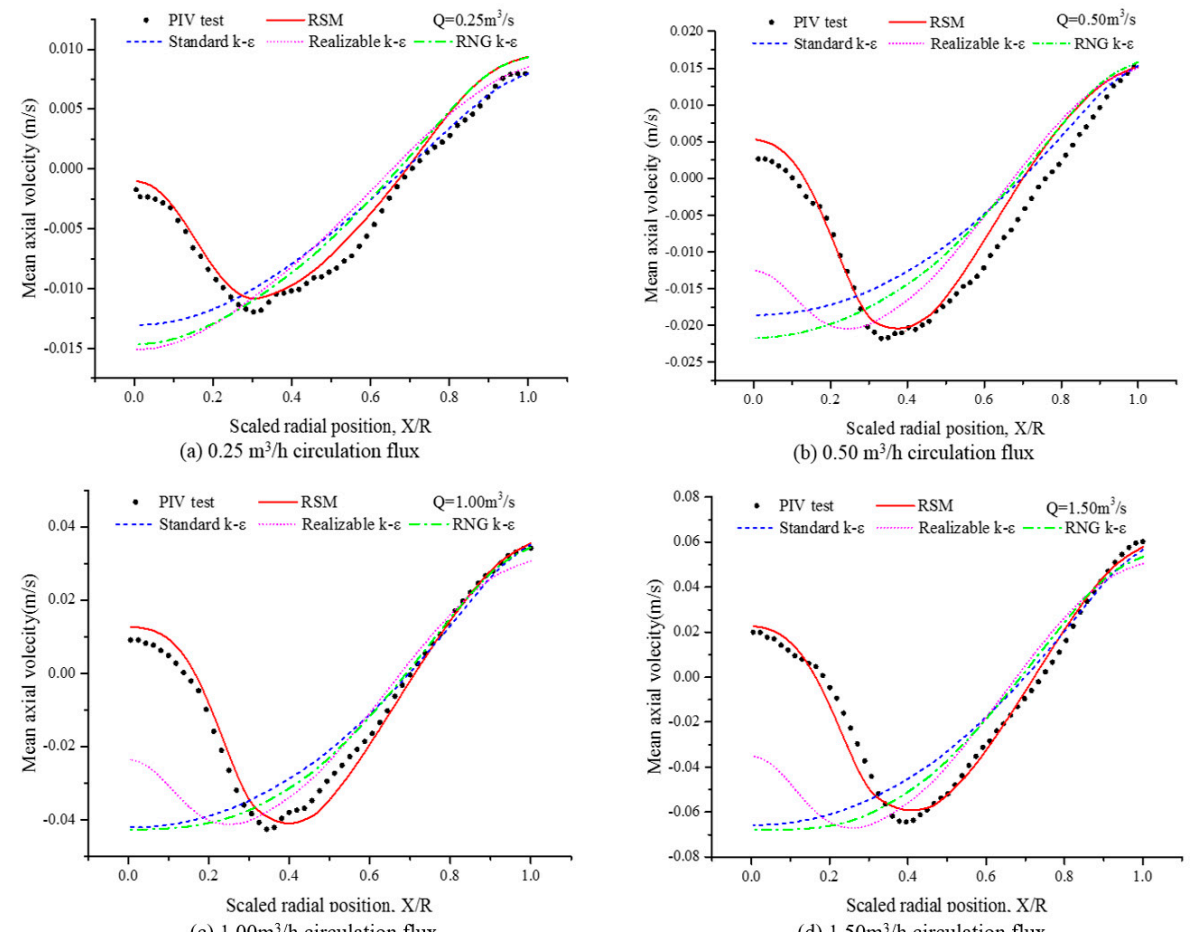

Figure 9. Axial velocity distribution calculated by turbulence models and PIV measurement along the horizontal direction, $300 \mathrm{~mm}$ high, and $10 \mathrm{~mm}$ from the axial section. 


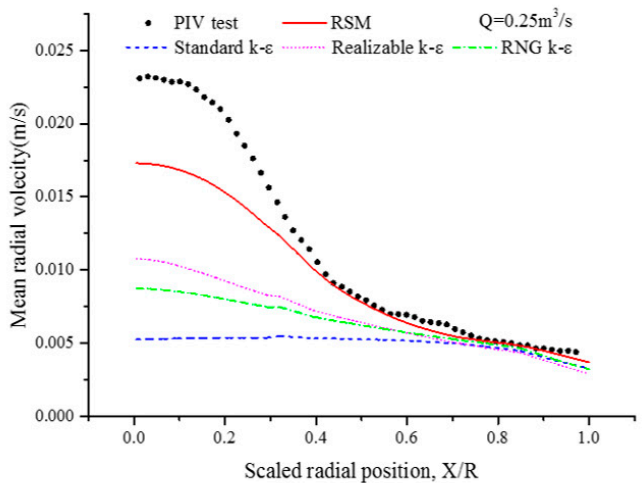

(a) $0.25 \mathrm{~m}^{3} / \mathrm{h}$ circulation flux

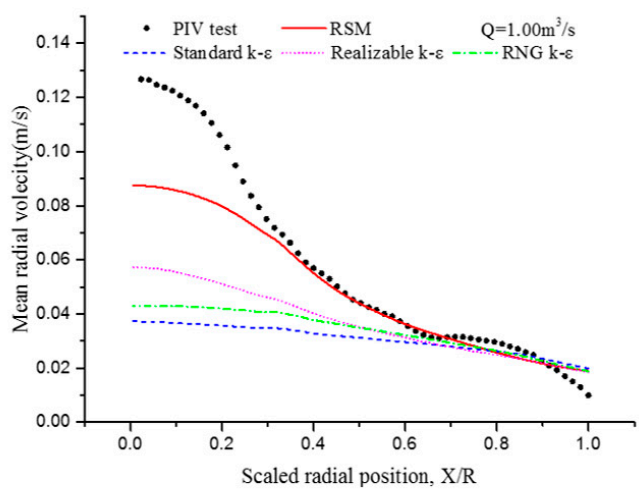

(c) $1.00 \mathrm{~m}^{3} / \mathrm{h}$ circulation flux

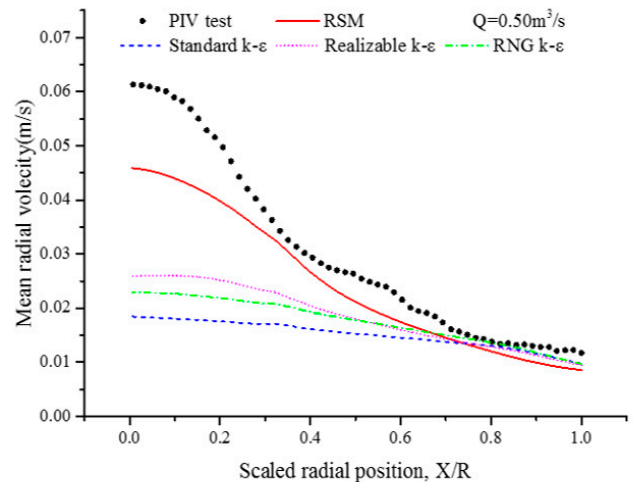

(b) $0.50 \mathrm{~m}^{3} / \mathrm{h}$ circulation flux

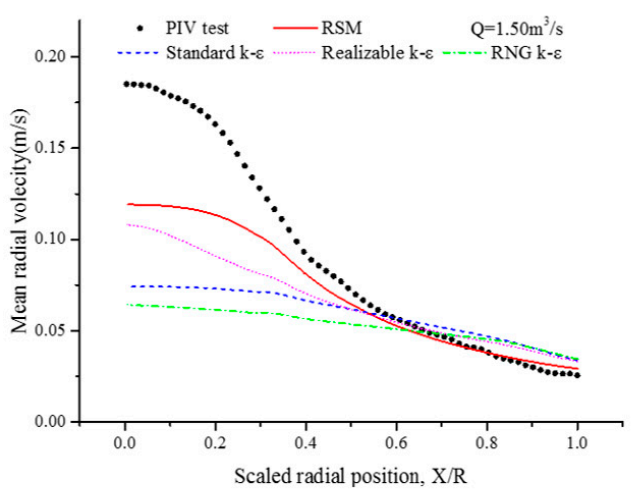

(d) $1.50 \mathrm{~m}^{3} / \mathrm{h}$ circulation flux

Figure 10. Radial velocity distribution calculated by turbulence models and PIV measurement along the horizontal direction, $300 \mathrm{~mm}$ high, and $10 \mathrm{~mm}$ from the axial section.

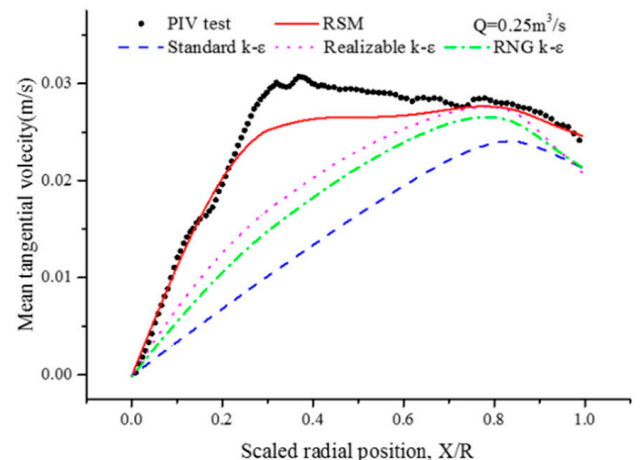

(a) $0.25 \mathrm{~m}^{3} / \mathrm{h}$ circulation flux

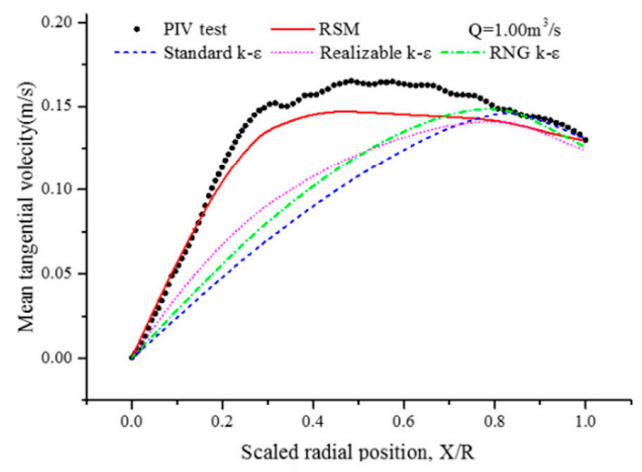

(c) $1.00 \mathrm{~m}^{3} / \mathrm{h}$ circulation flux

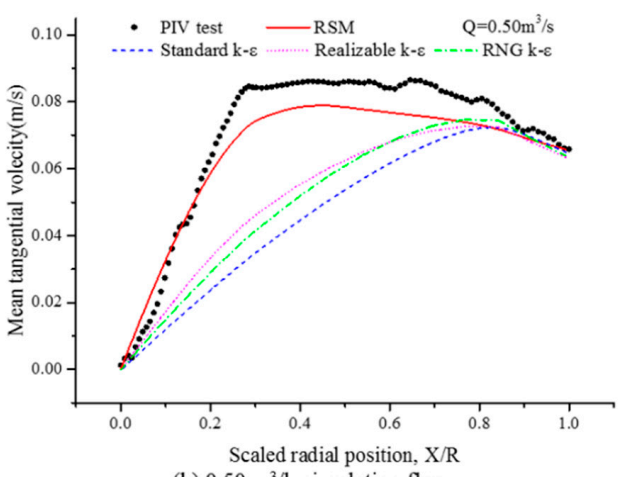

(b) $0.50 \mathrm{~m}^{3} / \mathrm{h}$ circulation flux

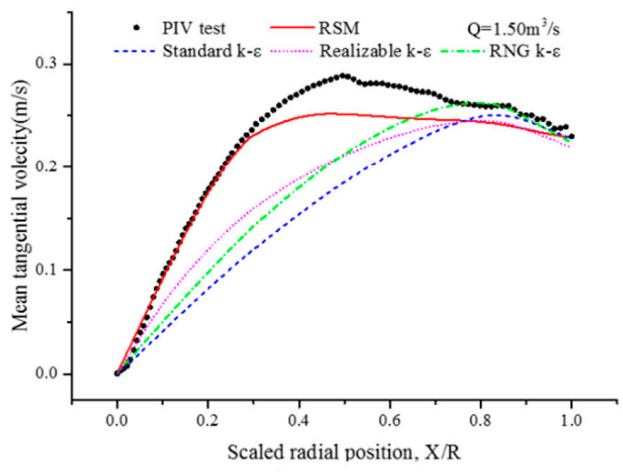

(d) $1.50 \mathrm{~m}^{3} / \mathrm{h}$ circulation flux

Figure 11. Tangential velocity distribution calculated by turbulence models and PIV measurement along the horizontal direction, $300 \mathrm{~mm}$ high. 
Table 6. Simulation model radial velocity absolute deviation from PIV measurement.

\begin{tabular}{ccccc}
\hline \multirow{2}{*}{ Flow Rate $\left(\mathbf{m}^{\mathbf{3}} \mathbf{h}\right)$} & \multicolumn{4}{c}{ Turbulence Model } \\
\cline { 2 - 5 } & RSM (\%) & Standard $\boldsymbol{k}-\boldsymbol{\varepsilon} \mathbf{( \% )}$ & Realizable $\boldsymbol{k - \varepsilon} \mathbf{~ ( \% )}$ & RNG $\boldsymbol{k - \varepsilon} \mathbf{~ ( \% )}$ \\
\hline 0.25 & 11.54 & 41.33 & 30.82 & 31.89 \\
0.50 & 18.23 & 38.42 & 30.50 & 31.29 \\
1.00 & 13.55 & 37.17 & 29.24 & 31.56 \\
1.50 & 15.86 & 32.06 & 25.20 & 36.54 \\
Mean absolute deviation & 14.79 & 37.25 & 28.94 & 32.82 \\
\hline
\end{tabular}

Table 7. Simulation model tangential velocity absolute deviation from PIV measurement.

\begin{tabular}{ccccc}
\hline \multirow{2}{*}{ Flow Rate $\left.\mathbf{( m}^{\mathbf{3}} \mathbf{h} \mathbf{h}\right)$} & \multicolumn{4}{c}{ Turbulence Model } \\
\cline { 2 - 5 } & $\mathbf{R S M} \mathbf{( \% )}$ & Standard $\boldsymbol{k - \varepsilon} \mathbf{( \% )}$ & Realizable $\boldsymbol{k - \varepsilon} \mathbf{( \% )}$ & RNG $\boldsymbol{k - \varepsilon} \mathbf{( \% )}$ \\
\hline 0.25 & 16.87 & 46.84 & 27.39 & 32.86 \\
0.50 & 13.87 & 37.74 & 27.88 & 30.79 \\
1.00 & 10.39 & 36.49 & 24.61 & 28.14 \\
1.50 & 12.77 & 34.60 & 24.60 & 26.61 \\
Mean absolute deviation & 13.48 & 38.92 & 26.12 & 29.60 \\
\hline
\end{tabular}

The $k-\varepsilon$ models are based on isotropic assumptions with semi-empirical formulas summarized from experimental results. However, the high-intensity swirling field has a large turbulent velocity gradient and fluctuating turbulent velocity, and hence is not even approximately isotropic. Therefore, $k-\varepsilon$ models are less effective, and the swirling flow field inside the flotation column cannot be correctly modelled. The RSM model is the most accurate model among the four models considered. This model abandons the isotropic assumption, considering laminar flow and Reynolds stress, and hence is suitable for anisotropic cases.

\section{Conclusions}

We performed numerical simulations and PIV measurements for a single-phase swirling field in a lab-scale FCSMC at four circulation fluxes $\left(0.25,0.50,1.00\right.$, and $\left.1.50 \mathrm{~m}^{3} / \mathrm{h}\right)$. Axial and radial velocity distributions were measured and modelled on the flotation column quasi-axial section. Experimental errors due to refraction at the free surface were avoided by adding an endoscopic lens to the CCD camera, inserting the speculum below the free surface, and allowing tangential velocity distribution to be measured on the indicated section. The RSM, standard $k-\varepsilon$ model, realizable $k-\varepsilon$ model, and RNG $k-\varepsilon$ model were used for CFD simulations for FCSMC single-phase swirling fields. Comparing the PIV experimental and simulation results and of the PIV, we made the following conclusions:

(1) Standard $k-\varepsilon$ model and RNG $k-\varepsilon$ model. Radial and tangential velocity distribution trends simulated by these models were consistent with PIV measurements, but axial velocity along the horizontal direction deviated widely from PIV results. Thus, axial errors were not considered. Mean absolute radial and tangential velocity deviations were $37.25 \%$ and $38.92 \%$, for the standard $k-\varepsilon$ model and slightly better for the RNG $k-\varepsilon$ model (32.82\% and $29.60 \%)$, respectively.

(2) RSM and realizable $k-\varepsilon$ model. Radial, tangential, and axial velocity distribution trends simulated by these models were all consistent with PIV measurements. Mean absolute axial, radial, and tangential velocity deviations were $17.36 \%, 14.79 \%$, and $13.48 \%$ for the RSM model, and $78.11 \%$, $28.94 \%$, and $26.12 \%$ for the realizable $k-\varepsilon$ model, respectively. Therefore, the RSM model exhibited superior performance, particularly in the axial direction.

(3) The $k-\varepsilon$ models [26] are based on isotropic assumptions with semi-empirical formulas summarized from experimental results. However, the internal flow field of the flotation column is a high-intensity vortex field with a large turbulent velocity gradient and fluctuating turbulent velocity, and hence is not approximately isotropic. The RSM model [27] abandons the isotropic assumption, considering laminar flow and Reynolds stress, and hence is suitable for anisotropic cases, especially the 
swirling flow field inside the flotation column. In fact, the RANS (Reynolds Averaged Navier-Stokes) model [28] does not distinguish between the size and direction of the vortex, and it is limited by the model conditions. Thus, it is difficult to get perfect results for complex problems such as an inverse pressure gradient numerical simulation. In addition, the RANS model relies on boundary conditions, so the calculations are very computationally dependent. DNS and even LES are good choices without considering computational costs and widespread adoption.

Author Contributions: Conceptualization, X.Y., Y.C.; methodology, S.M., L.W.; software, S.M., X.L.; validation, X.L.; investigation, H.Z.; data curation, S.M., X.Y.; writing-original draft preparation, S.M.; writing-review and editing, S.M., X.Y.; visualization, S.M., X.L.; and funding, Y.C., H.Z., L.W.

Funding: This research was funded by the National Natural Science Foundation of China (U1704252), the Fundamental Research Funds for the Central Universities (2017XKZD02) and the National Natural Science Foundation of China (51722405).

Conflicts of Interest: The authors declare no conflict of interest.

\section{References}

1. Jiongtian, L. Cyclonic-Static Micro-Bubble Floatation Apparatus and Method. U.S. Patent 6073775, 19 January 1999.

2. Tian, J.; Ni, L.; Song, T.; Olson, J.; Zhao, J. An overview of operating parameters and conditions in hydrocyclones for enhanced separations. Sep. Purif. Technol. 2018, 206, 268-285. [CrossRef]

3. Li, X.; Xu, H.; Liu, J.; Zhang, J.; Li, J.; Gui, Z. Cyclonic state micro-bubble flotation column in oil-in-water emulsion separation. Sep. Purif. Technol. 2016, 165, 101-106. [CrossRef]

4. Zhang, H.; Liu, J.; Wang, Y.; Cao, Y.; Ma, Z.; Li, X. Cyclonic-static micro-bubble flotation column. Miner. Eng. 2013, 45, 1-3. [CrossRef]

5. Guichao, W.; Linhan, G.; Subhasish, M.; Evans, G.M.; Joshi, J.B.; Songying, C. A review of CFD modelling studies on the flotation process. Miner. Eng. 2018, 127, 153-177.

6. Buchhave, P. Particle image velocimetry-Status and trends. Exp. Therm. Fluid Sci. 1992, 5, 586-604. [CrossRef]

7. Marins, L.P.M.; Duarte, D.G.; Loureiro, J.B.R.; Moraes, C.A.C.; Freire, A.P.S. LDA and PIV characterization of the flow in a hydrocyclone without an air-core. J. Pet. Sci. Eng. 2010, 70, 168-176. [CrossRef]

8. Cui, B.Y.; Wei, D.Z.; Gao, S.L.; Liu, W.G.; Feng, Y.Q. Numerical and experimental studies of flow field in hydrocyclone with air core. Trans. Nonferr. Met. Soc. China 2014, 24, 2642-2649. [CrossRef]

9. Yan, X.; Meng, S.; Wang, A.; Wang, L.; Cao, Y. Hydrodynamics and separation regimes in a cyclonic-static microbubble flotation column. Asia Pac. J. Chem. Eng. 2018, 13, e2185. [CrossRef]

10. Wang, A.; Yan, X.; Wang, L.; Cao, Y.; Liu, J. Effect of cone angles on single-phase flow of a laboratory cyclonic-static micro-bubble flotation column: PIV measurement and CFD simulations. Sep. Purif. Technol. 2015, 149, 308-314. [CrossRef]

11. Khan, Z.; Bhusare, V.H.; Joshi, J.B. Comparison of turbulence models for bubble column reactors. Chem. Eng. Sci. 2017, 164, 34-52. [CrossRef]

12. Zhang, M.; Li, T.; Wang, G. A CFD study of the flow characteristics in a packed flotation column: Implications for flotation recovery improvement. Int. J. Miner. Process. 2017, 159, 60-68. [CrossRef]

13. Yan, X.; Liu, J.; Cao, Y.; Wang, L. A single-phase turbulent flow numerical simulation of a cyclonic-static micro bubble flotation column. Int. J. Min. Sci. Technol. 2012, 22, 95-100. [CrossRef]

14. Swain, S.; Mohanty, S. A 3-dimensional Eulerian-Eulerian CFD simulation of a hydrocyclone. Appl. Math. Model. 2013, 37, 2921-2932. [CrossRef]

15. Azadi, M.; Azadi, M.; Mohebbi, A. A CFD study of the effect of cyclone size on its performance parameters. J. Hazard. Mater. 2010, 182, 835-841. [CrossRef] [PubMed]

16. Velarde, I.C.; Gallucci, F.; Annaland, M.V.S. Development of an endoscopic-laser PIV/DIA technique for high-temperature gas-solid fluidized beds. Chem. Eng. Sci. 2016, 143, 351-363. [CrossRef]

17. Helmi, I.C.V.A.; Gallucci, F.; van Sint Annaland, M. Hydrodynamics of dense gas-solid fluidized beds with immersed vertical membranes using an endoscopic-laser PIV/DIA technique. Chem. Eng. Sci. 2018, 182, 146-161. [CrossRef] 
18. Spalding, B.D. Lectures in Mathematical Models of Turbulence; Academic Press: Cambridge, MA, USA, 1972.

19. Victor Yakhot, S.A.O. Renormalization group analysis of turbulence: Basic theory. J. Sci. Comput. 1986, 1, 3-11. [CrossRef]

20. Peinke Moin, J.K.; Gerrit, O. Progress in Large Eddy Simulation of Turbulent Flows; AIAA Paper 97-15761; American Institute of Aeronautics and Astronautics: Reston, VA, USA, 1997.

21. Shih, T.H.; Liou, W.W.; Shabbir, A.; Yang, Z.; Zhu, J. A New Kappa-Epsilon Eddy Viscosity Model for high Reynolds Number Turbulent Flows-Model Development and Validation; Linthicum Heights Md Nasa Center for Aerospace Information |c: Linthicum Heights, MD, USA, 1995.

22. Orszag, S.A.; Yakhot, V.; Flannery, W.S.; Boysan, F.; Choudhury, D.; Maruzewski, J.; Patel, B. Renormalization Group Modeling and Turbulence Simulations; Elsevier: Amsterdam, The Netherlands, 1993.

23. Launder, B.E.; Reece, G.J.; Rodi, W. Progress in the development of a Reynolds-stress turbulence closure. J. Fluid Mech. 1975, 68, 537-566. [CrossRef]

24. Launder, B.E. Second-moment closure: Present ... and future? Int. J. Heat Fluid Flow 1989, 10, $282-300$. [CrossRef]

25. Gibson, M.M.; Launder, B.E. Ground effects on pressure fluctuations in the atmospheric boundary layer. J. Fluid Mech. 2006, 86, 491-511. [CrossRef]

26. Mendoza-Escamilla, V.X.; Alonzo-García, A.; Mollinedo, H.R.; González-Neria, I.; Yáñez-Varela, J.A.; Martinez-Delgadillo, S.A. Assessment of $k-\varepsilon$ models using tetrahedral grids to describe the turbulent flow field of a PBT impeller and validation through the PIV technique. Chin. J. Chem. Eng. 2018, 26, 942-956.

27. Khan, Z.; Joshi, J.B. Comparison of $\mathrm{k}-\varepsilon$, RSM and LES models for the prediction of flow pattern in jet loop reactor. Chem. Eng. Sci. 2015, 127, 323-333. [CrossRef]

28. Xiao, H.; Cinnella, P. Quantification of Model Uncertainty in RANS Simulations: A Review. Prog. Aerosp. Sci. 2019, 108, 1-31. [CrossRef]

(C) 2019 by the authors. Licensee MDPI, Basel, Switzerland. This article is an open access article distributed under the terms and conditions of the Creative Commons Attribution (CC BY) license (http://creativecommons.org/licenses/by/4.0/). 